\title{
Bounding the first exit from the basin: Independence times and finite-time basin stability
}

Paul Schultz, Frank Hellmann, Kevin N. Webster, and Jürgen Kurths

Citation: Chaos 28, 043102 (2018); doi: 10.1063/1.5013127

View online: https://doi.org/10.1063/1.5013127

View Table of Contents: http://aip.scitation.org/toc/cha/28/4

Published by the American Institute of Physics

\section{Articles you may be interested in}

On two diffusion neuronal models with multiplicative noise: The mean first-passage time properties

Chaos: An Interdisciplinary Journal of Nonlinear Science 28, 043103 (2018); 10.1063/1.5009574

Multistability and tipping: From mathematics and physics to climate and brain-Minireview and preface to the focus issue

Chaos: An Interdisciplinary Journal of Nonlinear Science 28, 033501 (2018); 10.1063/1.5027718

Topological characterization versus synchronization for assessing (or not) dynamical equivalence

Chaos: An Interdisciplinary Journal of Nonlinear Science 28, 045107 (2018); 10.1063/1.5011325

Phase reduction and synchronization of a network of coupled dynamical elements exhibiting collective oscillations

Chaos: An Interdisciplinary Journal of Nonlinear Science 28, 045103 (2018); 10.1063/1.5009669

Identifying influential nodes in complex networks: A node information dimension approach

Chaos: An Interdisciplinary Journal of Nonlinear Science 28, 043109 (2018); 10.1063/1.5030894

Identifying partial topology of complex dynamical networks via a pinning mechanism

Chaos: An Interdisciplinary Journal of Nonlinear Science 28, 043108 (2018); 10.1063/1.5009946

\section{PHYSICS TODAY}

\section{WHITEPAPERS}

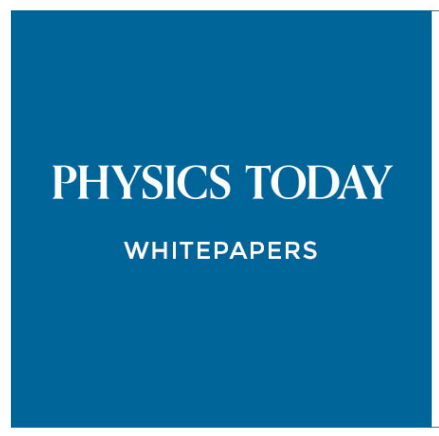

MANAGER'S GUIDE

Accelerate R\&D with

Multiphysics Simulation

\section{READ NOW}

PRESENTED BY

$\checkmark \subset \bigcirc M S O L$ 


\title{
Bounding the first exit from the basin: Independence times and finite-time basin stability
}

\author{
Paul Schultz, ${ }^{1,2, a)}$ Frank Hellmann, ${ }^{1}$ Kevin N. Webster, ${ }^{1}$ and Jürgen Kurths ${ }^{1,2,3,4}$ \\ ${ }^{1}$ Potsdam Institute for Climate Impact Research (PIK), Member of the Leibniz Association, P.O. Box 601203 , \\ D-14412 Potsdam, Germany \\ ${ }^{2}$ Department of Physics, Humboldt University of Berlin, Newtonstr. 15, 12489 Berlin, Germany \\ ${ }^{3}$ Institute for Complex Systems and Mathematical Biology, University of Aberdeen, Aberdeen AB24 $3 U E$, \\ United Kingdom \\ ${ }^{4}$ Department of Control Theory, Nizhny Novgorod State University, Gagarin Avenue 23, 606950 Nizhny \\ Novgorod, Russia
}

(Received 10 November 2017; accepted 22 March 2018; published online 4 April 2018)

\begin{abstract}
We study the stability of deterministic systems, given sequences of large, jump-like perturbations. Our main result is the derivation of a lower bound for the probability of the system to remain in the basin, given that perturbations are rare enough. This bound is efficient to evaluate numerically. To quantify rare enough, we define the notion of the independence time of such a system. This is the time after which a perturbed state has probably returned close to the attractor, meaning that subsequent perturbations can be considered separately. The effect of jump-like perturbations that occur at least the independence time apart is thus well described by a fixed probability to exit the basin at each jump, allowing us to obtain the bound. To determine the independence time, we introduce the concept of finite-time basin stability, which corresponds to the probability that a perturbed trajectory returns to an attractor within a given time. The independence time can then be determined as the time scale at which the finite-time basin stability reaches its asymptotic value. Besides that, finite-time basin stability is a novel probabilistic stability measure on its own, with potential broad applications in complex systems. Published by AIP Publishing. https://doi.org/10.1063/1.5013127
\end{abstract}

A central problem in the study of dynamical systems is quantifying the stability of an attractor. Looking at small perturbations leads to linear stability and Lyapunov exponents. Many applications instead require us to consider the chance that a large perturbation will not kick the system out of the basin of attraction called basin stability. ${ }^{1}$ If we add noise to the system, we can further ask about the expected time it takes for the system to first exit the basin.

In this work, we show how to use ideas from basin stability to study first exit times, if the noise is a sequence of large, but sufficiently rare jumps. We quantify precisely what sufficiently rare means and derive a bound for the first exit time distribution. Crucially, this bound is expressed in terms of quantities that can be evaluated efficiently for high-dimensional systems.

Thus, the results of this paper will have wide applications to high-dimensional systems with noise that can be approximated in the above way, e.g. power grids subjected to intermittent wind and solar power, neuronal networks, or ecosystems.

\section{INTRODUCTION}

A typical problem in the study of multi-stable dynamical systems is the stability of an attractor against perturbations. Especially finite perturbations potentially alter the timeasymptotic behaviour of a dynamical system and its

\footnotetext{
${ }^{\text {a)} E l e c t r o n i c ~ m a i l: ~ p s c h u l t z @ p i k-p o t s d a m . d e ~}$
}

macroscopic behaviour and explosive synchronisation transitions in networks of coupled oscillators ${ }^{2-4}$ may be mentioned exemplary. The various consequences of such phenomena for multistable biological systems are studied, for instance, in the field of network physiology $y^{5-7}$ but also find their application in technical fields like power system research..$^{8-10}$

For small perturbations, stability can be assessed in terms of asymptotic stability theory for linear systems, ${ }^{11}$ e.g., by calculating Lyapunov exponents.

On the other hand, for large perturbations, a typical approach is to assess stability by properties of the basin of attraction, for instance, their size. ${ }^{12-14}$ For this, several direct and sampling-based methods are available.

In particular, Lyapunov functions ${ }^{15-17}$ and related concepts like non-equilibrium potentials ${ }^{18,19}$ are powerful tools for studying basins of attraction. The existence of a global Lyapunov function ensures global stability against all perturbations.

The explicit construction of Lyapunov functions for a given system is a difficult problem in general. However, several numerical approaches for the computation of Lyapunov functions have been developed, including the SOS (sums of squares) method, ${ }^{20}$ the CPA (continuous piece-wise affine) method, ${ }^{21}$ radial basis functions, ${ }^{22}$ and the numerical solution of Zubov's equation. ${ }^{23}$ For a survey of these methods, see Ref. 17.

Direct methods, however, are typically not efficient for high-dimensional systems and yield conservative bounds on the attraction basin. ${ }^{24,25}$

Basin stability $\beta^{1,26,27}$ instead considers the probability that a system will return to an attractor following a large, jump-like perturbation. As other measures designed this way 
(e.g., Refs. 28 and 29), it has the advantage of allowing for efficient estimators by sampling the phase space and the trajectories directly. These estimators have a sampling error that is independent of the system dimension. Thus $\beta$ can be efficiently evaluated for high-dimensional systems and for dynamics where no analytic Lyapunov functions are known. ${ }^{26}$

In this paper, we study the behaviour of systems under repeated large perturbations. We answer the question of how rare perturbations need to be for basin stability to predict its probability to remain in the basin indefinitely.

To do so, we introduce the notion of the independence time of a system subject to a random perturbation. This captures the time the system takes to return to the attractor following a perturbation. An inescapable problem when studying the return of a system to an attractor lies in the fact that this return typically takes infinitely long and requires regularisation. ${ }^{30,31}$ Here we make use of the repeated perturbations to provide us with a meaningful regulator. We consider the system to have returned if the dynamics have erased the memory of the previous perturbation. More formally, the system has returned if its distribution following a perturbation is approximately equal to its distribution after a perturbation centered on the attractor. If this is the case, the states after subsequent perturbations, considered as random variables, are approximately statistically independent, and the probability to exit the basin factorizes.

To efficiently evaluate the independence time, we introduce the notion of finite-time basin stability $\beta(T)$. This is a finite-time horizon version of basin stability, corresponding to the probability that a system has returned to the attractor (according to a chosen criterion) in time $T$. By combining this with the return criterion required for independence time, we can give a lower bound for the independence time as the time when the finite-time basin stability approaches its asymptotic value. Furthermore, this enables us to derive an efficient estimator for a lower bound on the independence time for high-dimensional systems.

Given a set of perturbations that occur less frequently than the independence time, the probability to exit the basin of attraction is simply given in terms of the basin stability and the frequency of perturbations. This is particularly of interest if the asymptotic basin stability is close to unity for a given set of perturbations. Then, the independence time is the time interval that has to pass between perturbations to ensure that a sequence of such perturbations cannot destabilise the system.

\section{DEFINITIONS}

\section{A. The system}

We will consider an autonomous multi-stable dynamical system for which we can describe the dynamical evolution with a system of first-order ordinary differential equations, i.e.,

$$
\dot{x}=f(x),
$$

with states $x$ living in a phase space $X \subseteq \mathbb{R}^{n}$. We are interested in the case that the system has at least one stable fixed point, which, without loss of generality, we assume to be at the origin $x^{*}=0$, such that $f\left(x^{*}\right)=0$. We denote the basin of attraction of the origin as $B \subset X$. Accordingly, the basin stability $^{1}$ of the fixed point $x^{*}$ with respect to a probability density $\rho$ of perturbations is given by

$$
\beta:=\int_{X} \mathbf{1}_{B}(x) \rho(x) d x, \quad \beta \in(0 ; 1) .
$$

$\beta$ corresponds to the probability that the systeminitially at $x^{*}$-returns to the fixed point for a perturbation drawn from $\rho$. It is proportional to the basin volume if $\rho$ is chosen as a uniform probability density with large enough support.

We now subject the system of Eq. (1) to a possibly infinite sequence of jump perturbations, with magnitude $\Delta x_{i}$ drawn at random from a probability density $\rho(\Delta x)$ and starting at time $t=0$. We do not further specify the discrete times $t_{i}$ at which these perturbations occur, i.e., perturbations might appear regularly or according to some distribution. The minimum difference between subsequent perturbations will be denoted by $\Delta t=\min _{i}\left(t_{i}-t_{i+1}\right)$. Initialising the system at the attractor, this setup leads to the stochastic integral equation

$$
x(t)=\int_{0}^{t} \mathrm{~d} t^{\prime} f\left[x\left(t^{\prime}\right)\right]+\int_{0}^{t} \mathrm{~d} t^{\prime} \sum_{i=0}^{\infty} \Delta x_{i} \delta\left(t^{\prime}-t_{i}\right) .
$$

For convenience, we introduce the number of jumps $n(t)$ that have happened at a time $t$

$$
n(t)=\int_{0}^{t} \mathrm{~d} t \sum_{i=0}^{\infty} \delta\left(t-t_{i}\right) .
$$

We will be concerned with the remain probability

$$
P_{\text {remain }}(t, x(0)):=P\left(\forall 0 \leq t^{\prime} \leq t: x\left(t^{\prime}\right) \in B\right) .
$$

That is, the probability for the system to continuously remain within the basin of attraction. This is the cumulative probability of the complement of the distribution of the time of the first exit from the basin $p_{f e}(t)$ of the system

$$
P_{\text {remain }}(t, x(0))=1-\int_{0}^{t} p_{f e}\left(t^{\prime}\right) d t^{\prime} .
$$

Hence, all information about the exit times, including escape rates, is captured by it.

If the jumps in the system are sufficiently rare, we expect that the probability for a solution $x\left(t^{\prime}\right)$ to Eq. (3) to continuously remain in the basin of attraction up to time $t$ to be given by

$$
P_{\text {remain }}(t, x(0)) \approx \beta^{n(t)}
$$

that is, every perturbation counted by $n(t)$ has an equal and constant probability to leave the system within the basin of attraction (or for pushing it out).

In Sec. III, we will quantify what sufficiently rare means to achieve such a formula. Before, as an additional prerequisite, we turn to the definition of finite-time basin stability. 


\section{B. Finite-time basin stability}

Our analysis is based on the return times of perturbed states within the basin of attraction $B$ to the original attractor, i.e., to the fixed point $x^{*}$, defined through a time-tracking Lyapunov function. A Lyapunov function is a function $V(x)$ with negative orbital derivative, i.e., it decreases along trajectories of Eq. (1) and has a minimum or diverges to $-\infty$ at the fixed point $x^{*} .{ }^{11,15,16}$ Further, the fixed point is the only point in the basin for which it is minimal or negatively divergent. Given that such a function exists and $f(x)$ is sufficiently smooth, $x^{*}$ is asymptotically stable. A time-tracking Lyapunov function $V$ is defined on $B$ and satisfies the differential equation

$$
\frac{d}{d t} V(x(t))=-1,
$$

i.e., it strictly decreases along any trajectory of Eq. (3). It is straightforward to see that the values of such Lyapunov functions track the time. If $x(t)$ and $x\left(t^{\prime}\right)$ are two points on the same trajectory, then by integrating the defining equation above we have

$$
V(x(t))-V\left(x\left(t^{\prime}\right)\right)=t-t^{\prime} .
$$

To fully determine such a Lyapunov function we need to specify boundary conditions on a transverse surface $S$ (more precisely we require the surface to be noncharacteristic, see, e.g., Ref. 22). If we set $V(S)=0$, the timetracking Lyapunov function measures how long it has been since, or will be until the system crosses the surface $S$. We denote this Lyapunov function as $V_{S}$. We further assume that $S$ lies in $B$ entirely and set $V_{S}(x)=\infty$ for states $x \in X \backslash B$ outside the basin of attraction.

The set $S$ defines our return condition and the finite-time basin stability, given $\rho$ and $S$, is defined as

$$
\beta_{S}(T):=\int_{X} \mathbf{1}_{B}(x) \Theta\left(T-V_{S}(x)\right) \rho(x) d x \in(0 ; 1) .
$$

$\Theta$ denotes the Heaviside step-function. This is the probability that a trajectory, following a perturbation drawn from $\rho(x)$, will return to within $S$ around the attractor $x^{*}=0$ within time $T$. For well-behaved vector fields $f(x)$, one expects that $\lim _{T \rightarrow \infty} \beta_{S}(T)=\beta$. Note that the latter does not assume $S$ to be small but holds for all $S \in B$ even if they enclose almost the whole basin.

\section{APPROXIMATE INDEPENDENCE OF POST- PERTURBATION STATES}

We now turn to the key question: When do we consider the system to have returned? As noted above, we want "returned" to imply that, from the current position, the state following a perturbation of the system is statistically independent of the state after the preceding perturbation.

Therefore, we will consider the shifted perturbation distributions. Let us define a distance function $\imath\left(x, x^{\prime}\right)$ on the phase space as the $L^{1}$ norm of the difference of the shifted probability distributions $\rho_{x}(\cdot)=\rho(\cdot-x)$

$$
\imath\left(x, x^{\prime}\right)=\int_{X}\left|\rho_{x}(u)-\rho_{x^{\prime}}(u)\right| d u .
$$

This is visualised in Fig. 1, where the distance between $x$ and the fixed point $x^{*}$ is given by $l\left(x, x^{*}\right)$ as indicated by the shaded area. Note that for an arbitrary state vector $y$, $l(y, x)$ is a subadditive, symmetric, non-negative function of $x$ and vanishes for $x=y$; hence, it is a pseudometric on $X$. We will use the shorthand $\imath(x)=\imath\left(x, x^{*}\right)$ for the distance to the fixed point.

The expectation value of some observable $\chi(x)$ satisfying $|\chi(x)| \leq 1$ with respect to the two distributions $\rho$ and $\rho_{x}$ differs at most by $\imath(x)$

$$
\begin{gathered}
\left|\int_{X} \chi(u) \rho(u) d u-\int_{X} \chi(u) \rho_{x}(u) d u\right| \\
\leq \int_{X}|\chi(u)|\left|\rho(u)-\rho_{x}(u)\right| d u \\
\quad \leq \int_{X}\left|\rho(u)-\rho_{x}(u)\right| d u=\imath(x) .
\end{gathered}
$$

The probability to remain in the fixed point's basin of attraction after a perturbation originating at $x$ is given by the basin stability $\beta_{x}$ of the shifted probability density $\rho_{x}$

$$
\beta_{x}:=\int_{X} \mathbf{1}_{B}(u) \rho_{x}(u) d u \in[0 ; 1] .
$$

Both basin stability and finite-time basin stability are defined as the expectation value of the basin indicator function $\mathbf{1}_{B}$. Thus, in particular, we have that

$$
\left|\beta-\beta_{x}\right| \leq \imath(x) \text { and }\left|\beta(T)-\beta_{x}(T)\right| \leq \imath(x) .
$$

For a system Eq. (3) at a jump event $t_{i}$, the distribution of the state after the perturbation, which we denote $x\left(t_{i}^{+}\right)$, given the state before the jump $x\left(t_{i}^{-}\right)$is given by $\rho_{x\left(t_{i}^{-}\right)}$. Thus the difference in the probability to exit the basin from $x\left(t_{i}^{-}\right)$ as opposed to $x^{*}$ is bounded by $l\left(x\left(t_{i}^{-}\right)\right)$. The distance to the attractor in our metric $l$ is a meaningful measure for the return to the attractor. If it is small, the distribution after two different jump events, $t_{i}$ and $t_{j}$, is similar, and the jumps are approximately independent in the sense we require.

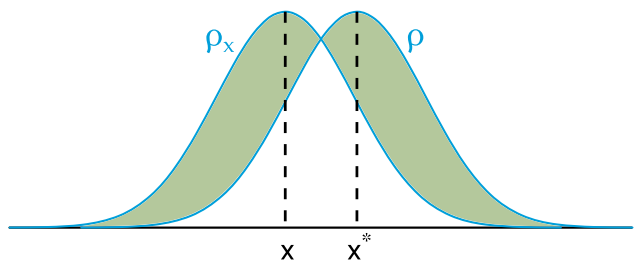

FIG. 1. Schematic representation of $l\left(x, x^{*}\right)$ : Imagine a one-dimensional system Eq. (1) with a fixed point $x^{*}$. The difference between the probability density $\rho$ centred at $x^{*}$ and the shifted density $\rho_{x}$ is then given by $\imath\left(x, x^{*}\right)$ (shaded green area) as defined in Eq. (11). 


\section{INDEPENDENCE TIMES}

To illustrate how independence can fail, consider Fig. 2(a). The figure shows the phase space of a damped driven pendulum, described by phase and frequency $x=(\phi, \omega)$. The shaded region is the basin of attraction of the fixed point $x^{*}$ at the origin. The shown trajectory is an example realisation of the deterministic dynamics being subject to jump perturbations [Eq. (3)] with $\Delta t$ chosen to be comparatively short. The perturbations are bounded in size, and the basin stability of the system is one. However, as they occur frequently, the system has no time to return to the attractor, leading to an eventual escape from the basin. After several jumps, $\beta_{x}$ starts being considerably smaller than $\beta$.

We can now combine the concepts introduced above to define a time that has to pass between subsequent perturbations, in order to prevent such a buildup.

For our definition of finite-time basin stability [cf. Eq. (10)], we have to specify a transverse surface $S$ for the timetracking Lyapunov function $V_{S}$. In particular, given an $\epsilon>0$, we choose $S$ such that $l(x)<\epsilon$ for all $x$ enclosed by $S$. Perturbations starting from the interior of $S$ are almost identical, with a deviation bounded by $\epsilon$.

The fact that $S$ is transverse, and its interior points satisfy $\imath(x)<\epsilon$, means that after the system enters $S, \imath(x(t))$ will never be larger than $\epsilon$ in the future.

Now given a threshold $\delta>0$, we define the independence time of a dynamical system as the time $T_{\text {ind }}(\epsilon, \delta)$ such that

$$
T_{\text {ind }}(\epsilon, \delta):=\inf \left\{T>0 \mid \beta-\beta_{S}(T) \leq \delta\right\} .
$$

That this time scale accurately quantifies independence of subsequent perturbations for system Eq. (3) is shown by the following result.

\section{A. Main result}

Given a sequence of perturbations drawn from $\rho$, occurring at times $t_{i}$ with minimum interval $\Delta t$ larger than the independence time $\Delta t>T_{\text {ind }}(\epsilon, \delta)$, the probability to
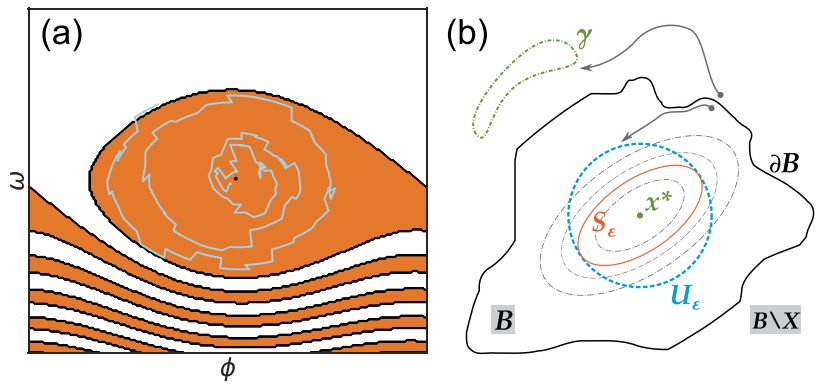

FIG. 2. (a) Example realisation of a swing equation [Eq. (27)] describing the evolution of phase $\phi$ and frequency $\omega$ dynamics of damped driven pendulum, discussed further in Sec. VI, subject to frequent, bounded jumps. The jump intervals are chosen to be comparatively short $(T=0.1)$; hence, the trajectory quickly escapes the corresponding basin of attraction (orange area). (b) Schematic picture of the basin of attraction $B$ with boundary $\partial B$ of the fixed point $x^{*}$ in a phase space $X$, visualising the relation of the sets $U_{\epsilon}$ and $S_{\epsilon}$ defined in Sec. V. In a multistable system, trajectories either approach the fixed point or other attractors, for instance, a limit cycle $\gamma$. remain within the basin of attraction, given that $x(0) \in S$, is bounded by

$$
P_{\text {remain }}(t, x(0)) \geq(\beta-\delta-\epsilon)^{n(t)}
$$

for all times $t>0$.

To show this, let us consider the perturbed system Eq. (3). At each jump event $t_{i}$, the conditional probability to not exit the basin of attraction is given by the shifted basin stability evaluated at the left limit $x_{i}$ of the trajectory before the jump

$$
P\left(x\left(t_{i}^{+}\right) \in B \mid x\left(t_{i}^{-}\right)=x_{i} \in B\right)=\beta_{x_{i}},
$$

where $t_{i}^{+}$and $t_{i}^{-}$denote the right, respectively, left limit of $t$ to the jump time $t_{i}$. Therefore, if we ensure that $\beta_{x_{i}}$ is close to $\beta$, we will also ensure that the perturbations are independent of each other in the sense we defined above.

Now given that the process is in $S$ before the jump at $t_{i}$, we want to understand what the probability is that it will return to $S$ before the next jump at $t_{i}$. If we started at the attractor rather than in $S$, this would be given by $\beta(\Delta t)$. The probability with respect to the shifted probability density thus differs from this at most by $\epsilon$. Assuming further that $\Delta t$ is larger than the independence time $T_{\text {ind }}$, Eq. (15) yields the lower bound

$$
\begin{aligned}
P\left(x\left(t_{i}^{-}\right) \in S \mid x\left(t_{i-1}^{-}\right) \in S\right) & \geq \beta(\Delta t)-\epsilon \\
& \geq \beta-\delta-\epsilon
\end{aligned}
$$

Thus, for a sequence of consecutive jumps counted by $n(t)$, we find

$$
\begin{aligned}
P_{\text {remain }}(t, x(0)) & \geq \prod_{i=1}^{n(t)} P\left(x\left(t_{i}^{-}\right) \in S \mid x\left(t_{i-1}^{-}\right) \in S\right) \geq \prod_{i=1}^{n(t)} \beta-\delta-\epsilon \\
& =(\beta-\delta-\epsilon)^{n(t)}
\end{aligned}
$$

The above formula applies as soon as the system enters the region bounded by $S$ once. Hence, if the stochastic process conditioned on staying in the basin of attraction has probability 1 of hitting $S$, Eq. (16) will also be the asymptotic form of the remain probability. Note also that the remain probability considers entire trajectories in the basin, not the probability to return there after having left.

The bound is necessarily not tight as it only considers trajectories that remain in the basin by returning to within $S$ before the next perturbation. We expect that for independence times corresponding to small $\delta$, this will be the dominant mechanism. For smaller times, there will be a nonnegligible contribution to the remain probability from jumps that cancel each other out.

\section{A PRACTICAL ESTIMATOR}

The above arguments establish a lower bound for the remain probability, but they do not provide an effective way to evaluate the quantities involved. The main difficulty in constructing an efficient estimator lies in evaluating the metric $l(x)$ and constructing a transverse return surface $S$ given an $\epsilon$. 
This problem simplifies considerably in the important special case that $\epsilon$ is chosen small enough that we only need to evaluate $l(x)$ close to the attractor. We now give an explicit formula based on the linearised dynamics for this case.

First let us consider $l(x)$. We Taylor expand $\rho$ around the origin to first order, and we find

$$
l(x) \simeq\|x\| \int_{X}\left\|\nabla \rho\left(x^{\prime}\right)\right\| d x^{\prime}=\frac{\|x\|}{C_{\rho}},
$$

defining a constant $C_{\rho}$ which is independent of the dynamics. It can be evaluated analytically for some common $\rho$, like uniform or Gaussian distributions, and numerically in general.

Thus, all points inside the sphere $U_{\epsilon}=\left\{x \mid\|x\|=\epsilon C_{\rho}\right\}$ satisfy $l(x) \leq \epsilon$. This sphere might not be transverse; hence, we are looking for a transverse surface $S_{\epsilon}$ of the timetracking Lyapunov function entirely contained within $U_{\epsilon}$. This is schematically illustrated in Fig. 2(b), where the relation between $U_{\epsilon}$ and $S_{\epsilon}$ is indicated for a fictional multistable system with a fixed point $x^{*}$ and corresponding basin $B$.

As we are in a neighbourhood of the fixed point, we can consider the linearised system associated with Eq. (1) given by

$$
\dot{x}(t)=J x(t) .
$$

If the Jacobian matrix $J$ is symmetric, then $U_{\epsilon}$ is transverse; we can choose $S_{\epsilon}=U_{\epsilon}$ and are done. To account for the general case, we can make use of quadratic Lyapunov functions $W(x)=x^{\dagger} L x$ for the linear system Eq. (21), satisfying $\dot{W}(x)=x^{\dagger} Q x$ with $Q$ symmetric and negative definite. Given $J$ and a choice of $Q$, we can find a Lyapunov function by solving the matrix equation

$$
J^{\dagger} L+L J=Q
$$

To find the maximum $|x|$ reached on the level set of $W(x)$, we differentiate $x^{2}$ in the direction parallel to the level set and look for extrema. Take a derivative $\partial_{v}=v \cdot \partial$. Then we require $\partial_{v} W(x)=0$ for the derivative to be tangential to the level set. An extremum on the level set thus satisfies the following set of equations:

$$
\begin{aligned}
\partial_{v} x^{2} & =2 v^{\dagger} x=0 \\
\forall v, \text { s.t. }: & v^{\dagger} L x+x^{\dagger} L v=2 v^{\dagger} L x=0,
\end{aligned}
$$

where we have used that $L$ is symmetric. We immediately see that for $L=1$, when our level sets are spheres, every point is an extremum. In general, it follows that as $x$ is orthogonal to all $v$, and the $v$ span the space orthogonal to $L x, x$ and $L x$ need to be parallel. Thus, the extrema are in the eigendirections of $L$. The maximum $x_{\text {max }}^{2}$ for a given level set is achieved in the eigendirection to the smallest eigenvalue $\lambda$; thus, the level set value is given by $W\left(x_{\text {max }}\right)=\lambda x_{\text {max }}^{\dagger} x_{\text {max }}$ $=\lambda\left[C_{\rho} l\left(x_{\text {max }}\right)\right]^{2}$. The largest level set contained in $U_{\epsilon}$ is thus given by $x^{\dagger} L x=\lambda\left(\epsilon C_{\rho}\right)^{2}$.

Therefore, the transverse surface $S_{\epsilon}$ is defined as

$$
S_{\epsilon}=\left\{x \mid x^{\dagger} L x=\lambda\left(\epsilon C_{\rho}\right)^{2}\right\} .
$$

The fact that we have $L$ on the left and $\lambda$ on the right shows that this relation does not depend on an overall scaling factor of the Lyapunov function. To make $S_{\epsilon}$ as large as possible, we want to make the ratios of the smallest eigenvalue of $L$ to the other ones, $\frac{\lambda_{i}}{\lambda}$, small. We leave the question, how to choose $Q$ such as to achieve this, open.

While direct Monte Carlo estimation of finite-time basin stability with the specified $S_{\epsilon}$ will lead to a valid independence time, the surface chosen will typically be far from optimal. The optimal surface $S_{\epsilon}^{\text {opt }}$ can be defined by taking the surface $S_{\epsilon}$ and evolving every point on it backwards in time until its $\imath$ distance to the attractor crosses $\epsilon$.

While this surface cannot be constructed explicitly in general, if $l(x)$ can be evaluated efficiently, we can evaluate the finite-time basin stability with respect to $S_{\epsilon}^{\text {opt }}$ by backtracking along the trajectories. In practice this means we start by generating trajectories that run until they hit $S_{\epsilon}$, guaranteeing that the $l(x)$ will never grow larger than $\epsilon$ again at later times, and then backtracking along the trajectory to find the first time where $l(x)>\epsilon$.

\section{A CONCRETE EXAMPLE}

In the following, we demonstrate the effective estimator for independence times, as well as the main result on remain probabilities, in a benchmark dynamical system.

For higher-dimensional systems, evaluating the Lyapunov function explicitly is not feasible. However, a sampling-based approach, analogous to basin stability estimations (e.g., Ref. 1), can be applied here.

The Monte-Carlo sampling procedure is as follows:

- Given a distribution $\rho$ and a tolerance $\epsilon$, determine $S_{\epsilon}$, for instance, using the method described in Sec. V.

- Sampling iteration:

1. Draw a random initial condition from $\rho_{x^{*}}$ centred at the fixed point.

2. Integrate the unperturbed system [Eq. (1)] until either it reaches $S_{\epsilon}$ or a cutoff time $T^{c}$ is reached. If it crosses $S_{\epsilon}$, record the time at which it does.

3. (optional) Backtrack along the trajectory to record the time at which $l(x)$ last crosses $\epsilon$.

The sampling step should be repeated for a sufficient ensemble of initial conditions to get significant statistics. Denote by $M_{T}$ the number of trajectories returning to $S_{\epsilon}$ within time $T$ or less and by $N$ the total number of trajectories sampled. Then, an estimator for the finite-time basin stability $\hat{\beta}(T)$ for $T<T^{c}$ is given by

$$
\hat{\beta}(T)=\frac{M_{T}}{N},
$$

with a standard error $e_{\hat{\beta}(T)}$ as

$$
e_{\hat{\beta}(T)}=\sqrt{\frac{\hat{\beta}(T)(1-\hat{\beta}(T))}{N}},
$$

since for a fixed $T$ we can regard this as a Bernoulli experiment, because trajectories either return or not. Note that if 
$\hat{\beta}(T) \approx 1$ or $\hat{\beta}(T) \approx 0$, more robust estimators are available. $^{32}$

Note that while the error decreases with the number of samples and does not depend on the dimensionality of the system, the time taken to evaluate a sample does depend on the system dimension at least linearly.

We will illustrate the numerical procedure by using the damped-driven pendulum as a benchmark system

$$
\begin{aligned}
& \dot{\phi}=\omega \\
& \dot{\omega}=p-\alpha \omega-k \sin \left(\phi+\arcsin \frac{p}{k}\right),
\end{aligned}
$$

with $p=1, \alpha=0.1$, and $k=8$. For this set of parameters, the system has two attractors, namely, a limit cycle and a fixed point $x^{*}=\left(\phi^{*}, \omega^{*}\right)=(0,0)$ at the origin. ${ }^{33}$

For illustrative purposes, we choose a distribution $\rho(x)$ to draw uniformly distributed perturbations at a point $x$ $=(\phi, \omega)$ from the box $R(\phi, \omega)=[\phi-\pi / 3 ; \phi+\pi / 3] \times[\omega$ $-5 ; \omega+5]$. This way, $R$ is almost entirely overlapping with the bulk of the basin of attraction of $x^{*}$ [cf. Fig. 2(a) for a schematic], such that we can expect $\beta$ to be close to 1 . Still, as we will see below, $\beta(T)$ can deviate strongly from $\beta$, especially for small $T$. To ensure sufficient statistics, we use a sample size of $N=20000$ points.

For this specific choice of $\rho$, we determine $S_{\epsilon}$ using Eq. (24) to be

$$
S_{\epsilon}=\left\{x \mid x^{\dagger} L^{\prime} x=\epsilon^{2}\right\},
$$

where $L^{\prime}=\frac{L}{\lambda C_{\rho}^{2}}$ is given by

$$
L^{\prime}=\left(\begin{array}{ll}
5.95152498 & 0.00838866 \\
0.00838866 & 0.74971598
\end{array}\right)
$$

Figure 3(a) summarises the results for the system Eq. (27). The horizontal blue line denotes the basin stability estimation $\hat{\beta}=0.9873 \pm 0.0025$ which is close to one as expected due to our choice of $R$. Indeed, beyond a certain time scale that depends on $\epsilon$, we observe that the finite-time basin stability curves approach the value of $\hat{\beta}$. From these points, we estimate the independence times $\hat{T}_{\text {ind }}(\epsilon, \delta)$ depicted in Fig. 3(b) using Eq. (15). As indicated by Fig. 3 (b), our results suggest that there is no significant dependence on the tolerance parameter $\delta$ for this particular system. Apparently, there is a rather sudden transition towards the value of $\hat{\beta}$ that cannot be resolved by the numerical differences of $T$-values. The crucial parameter here is $\epsilon$ determining the extent of the return set $S_{\epsilon}$. The logarithmic scale in Fig. 3(b) underlines that the independence time depends exponentially on the tolerance $\epsilon$ as the corresponding $S_{\epsilon}$ encloses the asymptotically stable fixed point $x^{*}$ even closer. Hence, the scaling $\hat{T}_{\text {ind }}(\epsilon, \delta) \sim \mathrm{e}^{\lambda \epsilon}$ seems to be determined by the real part $\lambda=-0.05$ of the two conjugate Jacobian eigenvalues of Eq. (27) linearised at $x^{*}$. This is indicated by the solid black line in Fig. 3(b) which has a slope of $\lambda$.

For comparison, Fig. 3(a) also depicts estimated $\beta(T)$ curves for the simulation of a high-dimensional system, namely, the Scandinavian power grid. The model details are outlined in Appendix A, see also the discussion in Hellmann
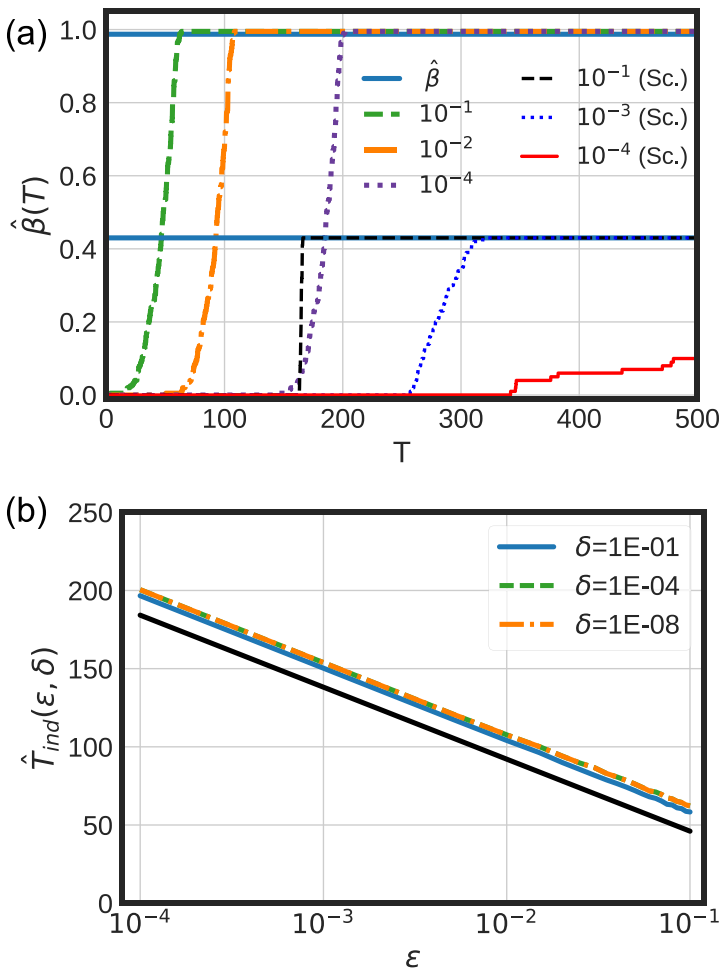

FIG. 3. (a) Estimated finite time basin stability $\hat{\beta}(T)$ curves [Eq. (10)] for selected $\epsilon$, simulated both for the pendulum model [Eq. (27)] and the Scandinavian power grid (prefix "Sc.," see also Appendix A). The value of $\epsilon$ is indicated in the legend. The solid blue lines give the respective basin stability [Eq. (2)] estimations $\hat{\beta}$. (b) The independence time $T_{\text {ind }}$ for various $\delta$ as a function of $\epsilon$ for the example of the pendulum model [Eq. (27)].

et al. ${ }^{29}$ The dynamical system resembles a network of secondorder Kuramoto oscillators ${ }^{34}$ [Eq. (A1)], analogous to the damped-driven pendulum in Eq. (27). Our numerical approach is as follows. First, we determine the synchronous phases [corresponding to a fixed point $x^{*}$ of Eq. (A1)] numerically and transform the coordinates such that $x^{*}$ is at the origin. We again choose a uniform distribution $\rho$, now with support $R(\phi, \omega)=[\phi-0.1 ; \phi+0.1] \times[\omega-0.1 ; \omega+0.1]$, encoding finite perturbations in any direction. This time, we resort to step 3 of the sampling iteration and evaluate $l$ directly along every trajectory to determine the return time to $U_{\epsilon}$. Note that this corresponds to calculate the overlap of two shifted hypercubes. We observe that the asymptotic basin stability $\beta$ is estimated to $\hat{\beta}=0.430 \pm 0.007$ for the above choice of $R$. Besides that, the estimated $\beta(T)$ curves of the high-dimensional system are qualitatively similar to the single pendulum. For smaller return sets, e.g., $\epsilon=10^{-6}$, there is further structure immanent to the $\beta(T)$ curve in the form of several plateaus for large return times. They correspond to steep transitions between the return times from different sets of initial conditions. Whether these features depend on the system size and how they relate to the network structure need to be further investigated in the future. Nevertheless, this example shows that the applicability of our approach is not restricted to lowdimensional systems. As we observed no qualitative differences between the model results, we however base the following discussion on the less complex pendulum model. 
We can now illustrate our result Eq. (16) for the probability to remain within the basin of attraction up to a certain time, given that we start in $S_{\epsilon}$ near the origin. For this, we simulate an ensemble of random processes by adding a jump process to the dynamics Eq. (27) [cf. Eq. (3)]. Explicitly, we choose 100 different time intervals $T$ between 10 and 400 time units such that after each interval a deviation is randomly selected according to a uniform distribution $\rho_{x^{\prime}}$ centred at the current state $x^{\prime}$ with a shifted domain $R$ as above. For each choice of $T$, we estimate the escape time distribution by recording the first time a trajectory jumps outside the origin's basin of attraction using an ensemble size of $N=1000$ trajectories. Denoting the number of trajectories with an escape time larger than $t$ by $N_{>}(t)$, we estimate the remain probability as $\hat{P}_{\text {remain }}=N_{>}(t) / N$. Then rewriting Eq. (16) as a per jump probability yields the following relation:

$$
p(t)=\hat{P}_{\text {remain }}^{\frac{1}{n(t)}} \geq \hat{\beta}-\epsilon-\delta,
$$

which we expect to hold if $T$ is larger than the corresponding independence time. For the system Eq. (27), the independence time for $\epsilon=10^{-1}$ is given by $\approx 60$, and for $\epsilon=10^{-2}$ it is $\approx 100$. We see in Fig. 4 that perturbations spaced 60 apart
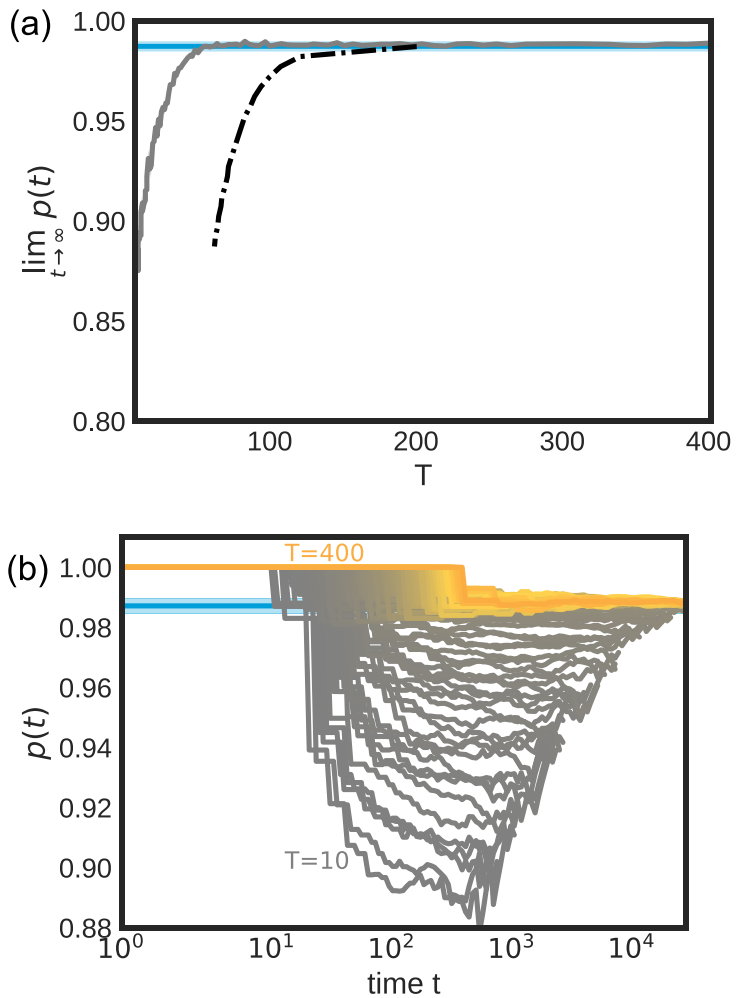

FIG. 4. In both figures, the horizontal blue line shows the estimate for the basin stability, and the shading indicates one standard error. (a) The solid line is the numerically determined asymptotic remain probability per jump event, $\lim _{t \rightarrow \infty} p(t)$ for perturbations that are at least $T$ apart, and the dasheddotted line shows the lower bound $\beta-\epsilon\left(T_{\text {ind }}\right)-\delta$ associated with an independence time $T_{\text {ind }}$, with $\delta=10^{-8}$. The function $\epsilon\left(T_{\text {ind }}\right)$ is the inverse of the estimated $\hat{T}_{\text {ind }}(\epsilon, \delta)$ at fixed $\delta$. As the dependence on $\delta$ is negligible [Fig. 3 (b)], we only depict the bound for $\delta=10^{-8}$. (b) The picture shows the remain probability $p(t)$ as a function of time $t$ given a particular perturbation interval $T$. Each curve corresponds to the fraction of trajectories that remain in the basin, perturbed at an interval $T$ which is indicated by the color progressing from grey to orange. cannot destabilise the system at a rate greater than $(\beta-0.1)$, and after $T=100$ we are within 0.01 of the basin stability asymptotic estimate (and thus close to its sampling error), as predicted. Further, by plotting the lower bound (for fixed $\delta=10^{-8}$ ) as a function of the independence time it is associated with, we see that our bound is satisfied across all times.

\section{DISCUSSION}

Just as for asymptotic basin stability, finite-time basin stability admits a simple and efficient sampling-based estimator that works for systems with a high number of dimensions. If the asymptotic basin stability is equal to one, this allows us to effectively guarantee, up to specified errors, that perturbations that occur at least the independence time apart cannot destabilise a system. We expect there to be a wide array of applications to the question, how rare large events have to be to not destabilise the system, which we intend to explore in future work.

We have also seen that the lower bound for which we developed the estimator is not sharp. This is entirely due to the estimate in Eq. (14), which bounds the shifted basin stability through the distance measure $\imath$. One challenge for future work is to develop and prove an effective estimator that can sidestep the use of $l$ and directly assess the escape probability.

More generally, we see under which conditions basin stability can be seen as the remain probability in the basin of attraction for systems subject to rare, strong events. Given the frequency of perturbations, basin stability completely determines the escape rate from the basin in this case.

One interesting analogue to our work is the study of the exit time distribution for basin escapes in systems subject to Levy noise. ${ }^{35,36}$ The type of stochastic process studied here, deterministic with interspersed jumps, can be used to approximate such Lévy processes in some asymptotic regime. ${ }^{37-39}$ We expect that the results of this paper can be used to develop estimators that can quantify when this asymptotic regime is reached. Consequently, it should lead to more efficient ways to perform an analysis as in Ref. 36 .

An open question for future work is to extend the notions discussed here to non-fixed point attractors. The main challenge here will lie in building a practical estimator that works.

\section{ACKNOWLEDGMENTS}

F.H. and J.K. acknowledge the support of BMBF, CoNDyNet, FK. 03SF0472A. K.W. was supported by the European Commission's Marie Curie Fellowship MALCOD (Grant No. 660616). This work was supported by the Volkswagen Foundation (Grant No. 88462). Funded by the Deutsche Forschungsgemeinschaft (DFG, German Research Foundation)-KU 837/39-1/RA 516/13-1. All authors gratefully acknowledge the European Regional Development Fund (ERDF), the German Federal Ministry of Education and Research, and the Land Brandenburg for supporting this project by providing resources on the high performance computer system at the Potsdam Institute for Climate Impact Research. 


\section{APPENDIX A: THE SCANDINAVIAN POWER GRID MODEL}

The Scandinavian dataset has been originally published in Menck et al. ${ }^{8}$ The (extra-)high voltage network contains $n=236$ nodes (plants, substations) and 320 links (transmission lines) which corresponds to an average node degree $\bar{k}$ of $\bar{k}=2.7$, characterising the sparse network structure. ${ }^{40}$ Commonly, the synchronisation dynamics are modelled by a network of coupled second-order Kuramoto oscillators ${ }^{34}$ characterised by their phase $\theta_{k}$ and instantaneous frequency $\omega_{k}$. The fixed point is shifted to the origin by defining the phases relative to $x^{*}=\left(\phi_{1}^{*}, \ldots, \phi_{n}^{*}, \omega_{1}^{*}, \ldots, \omega_{n}^{*}\right)$.

The dynamical system reads

$$
\begin{aligned}
\dot{\theta}_{k} & =\omega_{k} \\
\dot{\omega}_{k} & =P_{k}-\alpha \omega_{k}-\sum_{j=1}^{n} A_{k j} \sin \left(\theta_{k}+\phi_{k}^{*}-\theta_{j}-\phi_{j}^{*}\right) .
\end{aligned}
$$

Here, each node represents a region of net power input (positive natural frequency $P_{k}=1$ ) or net consumption $\left(P_{k}=-1\right)$. The input powers are randomly assigned to the nodes. The network topology is encoded by a weighted adjacency matrix with entries $A_{k j}$ according to the transmission capacities. The link weights are functions of the link length; in our dimensionless units their average is $\left\langle A_{k j}\right\rangle_{k j}=6$. The damping parameter $\alpha$ is taken to be $\alpha=0.1$.

${ }^{1}$ P. J. Menck, J. Heitzig, N. Marwan, and J. Kurths, "How basin stability complements the linear-stability paradigm,” Nat. Phys. 9, 89-92 (2013).

${ }^{2}$ J. Gómez-Gardeñes, S. Gómez, A. Arenas, and Y. Moreno, "Explosive synchronization transitions in scale-free networks," Phys. Rev. Lett. 106, 128701 (2011).

${ }^{3} \mathrm{X}$. Zhang, X. Hu, J. Kurths, and Z. Liu, "Explosive synchronization in a general complex network," Phys. Rev. E 88, 010802(R) (2013).

${ }^{4}$ X. Zhang, A. Pikovsky, and Z. Liu, "Dynamics of oscillators globally coupled via two mean fields," Sci. Rep. 7, 2104 (2017).

${ }^{5}$ A. Bashan, R. P. Bartsch, J. W. Kantelhardt, S. Havlin, and P. C. Ivanov, "Network physiology reveals relations between network topology and physiological function," Nat. Commun. 3, 702 (2012).

${ }^{6}$ R. P. Bartsch, K. K. L. Liu, A. Bashan, and P. C. Ivanov, "Network physiology: How organ systems dynamically interact," PLoS One 10, e0142143 (2015).

${ }^{7}$ K. K. L. Liu, R. P. Bartsch, A. Lin, R. N. Mantegna, and P. C. Ivanov, "Plasticity of brain wave network interactions and evolution across physiologic states," Front. Neural Circuits 9, 62 (2015).

${ }^{8}$ P. J. Menck, J. Heitzig, J. Kurths, and H.-J. Schellnhuber, "How dead ends undermine power grid stability," Nat. Commun. 5, 3969 (2014).

${ }^{9}$ K. Schmietendorf, J. Peinke, R. Friedrich, and O. Kamps, "Self-organized synchronization and voltage stability in networks of synchronous machines,” Eur. Phys. J.: Spec. Top. 223(12), 1-9 (2014).

${ }^{10}$ H. Kim, S. H. Lee, and P. Holme, "Building blocks of the basin stability of power grids," Phys. Rev. E 93, 062318 (2016).

${ }^{11}$ A. M. Lyapunov, "Problème Général de la Stabilité du Mouvement," Ann. Fac. Sci. Toulouse: Math. 2, 203-474 (1907).

${ }^{12}$ D. A. Wiley, S. H. Strogatz, and M. Girvan, "The size of the sync basin," Chaos 16, 015103 (2006).

${ }^{13}$ V. V. Klinshov, V. I. Nekorkin, and J. Kurths, "Stability threshold approach for complex dynamical systems," New J. Phys. 18, 013004 (2015).
${ }^{14}$ C. Mitra, J. Kurths, and R. V. Donner, "An integrative quantifier of multistability in complex systems based on ecological resilience," Sci. Rep. 5, 16196 (2015).

${ }^{15}$ W. Hahn, "Über die Anwendung der Methode von Ljapunov auf Differenzengleichungen," Math. Ann. 136, 430-441 (1958).

${ }^{16} \mathrm{M}$. Malisoff and F. Mazenc, Constructions of Strict Lyapunov Functions, Communications and Control Engineering, 1st ed. (Springer, London, 2009), p. XVI, 386.

${ }^{17} \mathrm{P}$. Giesl and S. Hafstein, "Review on computational methods for Lyapunov functions," Discrete Contin. Dyn. Syst., Ser. B 20, 2291-2331 (2015).

${ }^{18} \mathrm{R}$. Graham and T. Tél, "Existence of a potential for dissipative dynamical systems," Phys. Rev. Lett. 52, 9-12 (1984).

${ }^{19}$ R. Graham, A. Hamm, and T. Tél, "Nonequilibrium potentials for dynamical systems with fractal attractors or repellers," Phys. Rev. Lett. 66, 3089-3092 (1991).

${ }^{20} \mathrm{P}$. Parrilo, "Structured semidefinite programs and semialgebraic geometry methods in robustness and optimization," Ph.D. thesis (California Institute of Technology, Pasadena, CA, 2000).

${ }^{21}$ S. Hafstein, "A constructive converse Lyapunov theorem on exponential stability," Discrete Contin. Dyn. Syst. 10, 657-678 (2004).

${ }^{22} \mathrm{P}$. Giesl, Construction of Global Lyapunov Functions Using Radial Basis Functions, Lecture Notes in Mathematics Vol. 1904 (Springer, Berlin, 2007).

${ }^{23}$ F. Camilli, L. Grüne, and F. Wirth, "A generalization of Zubov's method to perturbed systems," SIAM J. Control Optim. 40, 496-515 (2001).

${ }^{24}$ H.-D. Chiang, Direct Methods for Stability Analysis of Electric Power Systems (John Wiley \& Sons, Inc., Hoboken, NJ, USA, 2010).

${ }^{25}$ A. Gajduk, M. Todorovski, and L. Kocarev, "Stability of power grids: An overview," Eur. Phys. J.: Spec. Top. 223, 2387-2409 (2014).

${ }^{26}$ P. Schultz, P. J. Menck, J. Heitzig, and J. Kurths, "Potentials and limits to basin stability estimation," New J. Phys. 19, 023005 (2017).

${ }^{27}$ C. Mitra, A. Choudhary, S. Sinha, J. Kurths, and R. V. Donner, "Multiplenode basin stability in complex dynamical networks," Phys. Rev. E 95, 032317 (2017).

${ }^{28}$ G. Rega and S. Lenci, "Identifying, evaluating, and controlling dynamical integrity measures in non-linear mechanical oscillators," Nonlinear Anal.: Theory, Methods Appl. 63, 902-914 (2005).

${ }^{29}$ F. Hellmann, P. Schultz, C. Grabow, J. Heitzig, and J. Kurths, "Survivability of deterministic dynamical systems," Sci. Rep. 6, 29654 (2016).

${ }^{30}$ T. Kittel, J. Heitzig, K. Webster, and J. Kurths, "Timing of transients: Quantifying reaching times and transient behavior in complex systems," New J. Phys. 19, 083005 (2017).

${ }^{31}$ C. Mitra, T. Kittel, A. Choudhary, J. Kurths, and R. V. Donner, "Recovery time after localized perturbations in complex dynamical networks," New J. Phys. 19, 103004 (2017).

${ }^{32}$ A. Agresti and B. A. Coull, "Approximate is better than "exact" for interval estimation of binomial proportion," Am. Stat. 52, 119-126 (1998).

${ }^{33}$ Note that we applied a phase shift of $\arcsin (p / k)$ to set the fix point to the origin.

${ }^{34}$ F. A. Rodrigues, T. K. D. Peron, P. Ji, and J. Kurths, "The Kuramoto model in complex networks," Phys. Rep. 610, 1-98 (2016).

${ }^{35}$ L. Serdukova, Y. Zheng, J. Duan, and J. Kurths, "Stochastic basins of attraction for metastable states," Chaos 26, 073117 (2016).

${ }^{36}$ L. Serdukova, Y. Zheng, J. Duan, and J. Kurths, "Metastability for discontinuous dynamical systems under Lévy noise: Case study on Amazonian vegetation," Sci. Rep. 7, 9336 (2017)

${ }^{37}$ P. Imkeller and I. Pavlyukevich, "Lévy flights: Transitions and metastability,” J. Phys. A: Math. Gen. 39, L237-L246 (2006).

${ }^{38}$ I. Pavlyukevich, "Lévy flights, non-local search and simulated annealing," J. Comput. Phys. 226, 1830-1844 (2007).

${ }^{39}$ I. Pavlyukevich, "Cooling down Lévy flights," J. Phys. A: Math. Theor. 40, 12299-12313 (2007).

${ }^{40}$ P. Schultz, J. Heitzig, and J. Kurths, "A random growth model for power grids and other spatially embedded infrastructure networks," Eur. Phys. J.: Spec. Top. 223, 2593-2610 (2014). 